\title{
DÜBLIN
}

Technological University Dublin

ARROW@TU Dublin

\section{Planar Dual-mode MIMO Antenna with Enhanced Bandwidth}

\author{
Adam Narbudowicz \\ Technological University Dublin, adam.narbudowicz@mydit.ie \\ Slawomir Koziel \\ Reykjavik University \\ Max Ammann \\ Technological University Dublin, max.ammann@tudublin.ie
}

See next page for additional authors

Follow this and additional works at: https://arrow.tudublin.ie/engscheleart

Part of the Electromagnetics and Photonics Commons

\section{Recommended Citation}

A. Narbudowicz, S. Koziel, M. J. Ammann, and D. Heberling, (2015) Planar Dual-mode MIMO Antenna with Enhanced Bandwidth, IEEE AP-S International Symposium on Antennas and Propagation, Vancouver, Canada, Institute of Electronic \& Electrical Engineers, pp. TU-A5.1P.2: MIMO Antennas, 3308, 19/07/2015.

This Conference Paper is brought to you for free and open access by the School of Electrical and Electronic Engineering at ARROW@TU Dublin. It has been accepted for inclusion in Conference papers by an authorized administrator of ARROW@TU Dublin. For more information, please contact arrow.admin@tudublin.ie, aisling.coyne@tudublin.ie,gerard.connolly@tudublin.ie. Funder: Irish Research Council

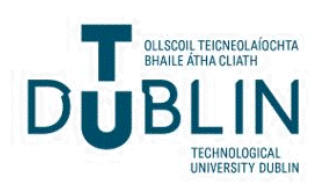


Authors

Adam Narbudowicz, Slawomir Koziel, Max Ammann, and Dirk Heberling

This conference paper is available at ARROW@TU Dublin: https://arrow.tudublin.ie/engscheleart/238 


\title{
Planar Dual-mode MIMO Antenna with Enhanced Bandwidth
}

\author{
Adam Narbudowicz ${ }^{1,2}$, Slawomir Koziel ${ }^{3}$, Max J. Ammann ${ }^{1}$, and Dirk Heberling ${ }^{2}$ \\ ${ }^{1}$ Antenna \& High Frequency Research Centre \\ Dublin Institute of Technology \\ Dublin, Ireland \\ \{adam.narbudowicz; \\ max.ammann\}@dit.ie \\ ${ }^{2}$ Institute of High Frequency Technology \\ RWTH Aachen University \\ Aachen, NRW, Germany \\ heberling@ihf.rwth-aachen.de \\ ${ }^{3}$ Engineering Optimization \\ $\&$ Modeling Center \\ Reykjavik University \\ Reykyavik, Iceland \\ koziel@ru.is
}

\begin{abstract}
The paper proposes a dual-port patch antenna for MIMO applications, optimized using surrogate-based optimization techniques. The antenna uses two resonant modes $\left(T M_{100}\right.$ and $\left.T M_{200}\right)$ to generate two independent radiation patterns. To enhance the bandwidth of $\mathbf{T} \mathbf{M}_{200}$ mode, the patch ground plane was realized as a meshed surface. The proposed approach provides $97 \mathrm{MHz}$ of bandwidth, while preserving a low profile of $1.5 \mathrm{~mm}$ between the patch and the groundplane $\left(\varepsilon_{\mathrm{r}}=\right.$ 3.5). The numerical optimization involved 12 adjustable geometry parameters.
\end{abstract}

\section{INTRODUCTION}

MIMO antenna systems are becoming more and more popular, increasing communication capacity of radio communication systems. Although initially, such systems relied on a number of individual antennas, the requirement for greater integration brought attention to more compact solutions [1-3]: a single antenna with multiple ports, where each port is able to create a different radiation pattern, hence supporting uncorrelated signals. Among those, multi-mode patch antennas were investigated in [3]. These structures involve multiple patches of various sizes, each operating in a different resonance mode (here $\mathrm{TM}_{100}$ and $\mathrm{TM}_{200}$ ). Such antennas are simple and low profile, however higher resonance modes would produce narrower bandwidth, limiting antenna operability.

In this paper we propose a dual-mode patch antenna, which has been designed to increase the bandwidth of the $\mathrm{TM}_{200}$ mode. This was achieved by replacing a solid ground plane with a grid. To maximize the antenna performance, numerical optimization of the high-fidelity electromagnetic (EM) simulation model of the antenna structure implemented in CST Microwave Studio was conducted. Because of a large number of geometry parameters to be simultaneously adjusted and a high computational cost of the EM analysis, surrogate-based optimization techniques have been utilized involving, among others, a faster version of the EM model with reduced accuracy. The final design obtained at a reasonable CPU cost provides 97 $\mathrm{MHz}$ of bandwidth.

\section{ANTENNA DESCRIPTION}

The antenna operates at $5.6 \mathrm{GHz}$ and consists of two patches (see Fig. 1), realized using three layers of Taconic ${ }^{\mathrm{TM}}$ RF-35 substrate (each layer being $1.5 \mathrm{~mm}$ high) and four metallization layers.

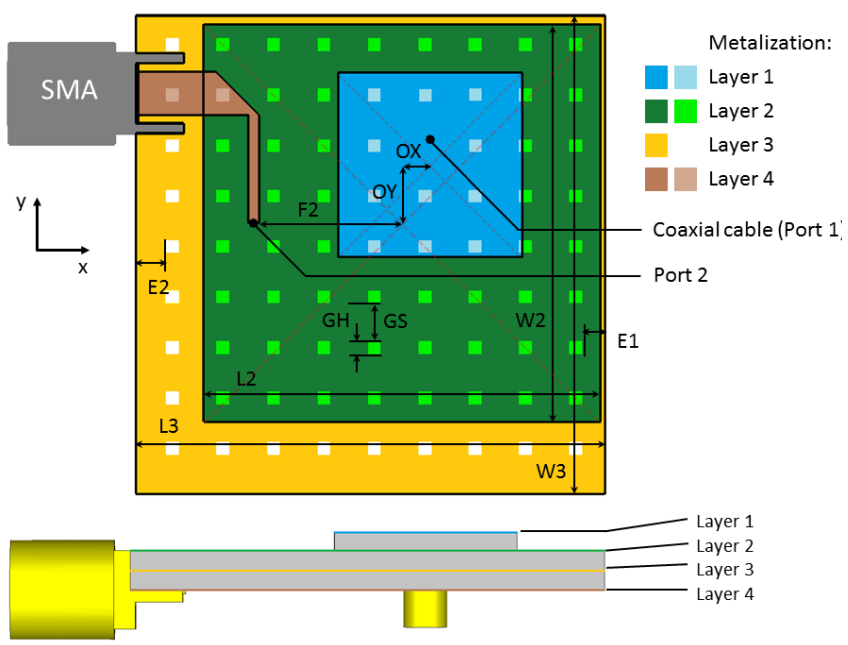

Fig. 1. The structure of the proposed antenna. All marked parameters have been utilized in the optimization process.

The top-most Layer 1 is a $\mathrm{TM}_{100}$ resonator, which is fed via a coaxial cable. Its dimensions are $W_{1}=L_{1}=14.5 \mathrm{~mm}$, and the feed is located $2 \mathrm{~mm}$ from its center. Those dimensions are kept fixed throughout the optimization process.

The larger patch, operating at $\mathrm{TM}_{200}$ resonance mode, is located on Layer 2. It serves as a ground plane for the $\mathrm{TM}_{100}$ patch and is therefore kept as a solid conductor to ensure good isolation between the two modes. Its dimensions are $W_{2}$ and $L_{2}$. To simplify optimization, the larger patch is fed directly from a discrete port located at a distance $F_{2}$ from the center, between Layers 2 and 3. The port impedance is one of the optimization parameters $Z_{i n}$. Parameters $O X$ and $O Y$ describe the horizontal and vertical offset between the centers of the two patches, respectively.

The ground plane of the $\mathrm{TM}_{200}$ patch is realized on Layer 3 as a mesh, with square slots of the width $G H$, separated by a distance $G S$. This is to increase the bandwidth of the $\mathrm{TM}_{200}$ resonance and was subjected to the optimization process. The areas at distances $E_{1}$ and $E_{2}$ from the edges is solid, i.e. without slots.

The lowest Layer 4 accommodates the impedance transformer and the SMA connector. These components were 
not excited, as the larger patch was fed from the discrete port 2 , located between Layers 2 and 3. However, they were utilized at a later stage of antenna's development and were included in the optimization, as their presence is expected to influence the radiation performance.

\section{OPTIMIZATION}

The high-fidelity EM simulation antenna model $\boldsymbol{R}_{f}$ is implemented in CST MWS (time domain solver) with $\sim 3,000,000$ mesh cells. Its simulation time is about 90 minutes on an 8-core 2.1 GHz Intel Xeon CPU with 64GB RAM. The goal is to solve the following maximization problem

$$
\boldsymbol{x}=\arg \max _{\boldsymbol{x}} B W\left(\boldsymbol{R}_{f}(\boldsymbol{x})\right)
$$

where $B W\left(\boldsymbol{R}_{f}(\boldsymbol{x})\right)$ is the antenna bandwidth, whereas $\boldsymbol{x}$ is a vector of geometry parameters as described in Section II. Because $\boldsymbol{R}_{f}$ is very expensive, the optimization process is conducted using its faster version, the low-fidelity model $\boldsymbol{R}_{c}$, which is the same as $\boldsymbol{R}_{f}$ but with relaxed convergence criteria ($15 \mathrm{~dB}$ accuracy instead of $-35 \mathrm{~dB}$ for $\boldsymbol{R}_{f}$; simulation time 25 minutes). The low-fidelity model overestimates the bandwidth but it is well correlated with $\boldsymbol{R}_{f}$.

The optimization process has three stages:

1. Direct optimization of $\boldsymbol{R}_{c}$ to find its approximate optimum;

2. Surrogate-based optimization of $\boldsymbol{R}_{f}$ using local response surface approximation (RSA) models constructed from sampled $\boldsymbol{R}_{c}$ data and space mapping correction [4].

3. Design tuning through sequential approximate optimization of $\boldsymbol{R}_{f}$, also based on its local RSA models.

Stage 1 of the process gives a good initial design (pattern search [5] is utilized due to numerical noise contained in $\boldsymbol{R}_{c}$ and resulting from limited simulation accuracy). The RSA models utilized in Stages 2 and 3 are second-order polynomials (without mixed terms) established using star-distribution training set $(2 n+$ 1 points with $n$ being the number of design variables) [5]. Stage 1 required 200 evaluations of $\boldsymbol{R}_{c}$; Stage 2 required 5 iterations of setting up the RSA model and its optimization (cost: $25 \times \boldsymbol{R}_{c}$ and $1 \times \boldsymbol{R}_{f}$ per iteration); Stage 3 required 2 iterations (cost $25 \times \boldsymbol{R}_{f}$ per iteration). Thus, the total optimization cost was corresponding to about 140 evaluations of the high-fidelity EM model ( 200 hours of CPU time).

\section{RESUlTS}

The optimized antenna parameters are as follows: $L_{2}=31.2 \mathrm{~mm} ; W_{2}=31.2 \mathrm{~mm} ; L_{3}=36.9 \mathrm{~mm} ; W_{3}=37.6 \mathrm{~mm}$; $E_{1}=1.7 \mathrm{~mm} ; \quad E_{2}=0.2 \mathrm{~mm} ; \quad G H=1.2 \mathrm{~mm} ; \quad G S=2.8 \mathrm{~mm}$; $O X=2.2 \mathrm{~mm} ; O Y=4.6 \mathrm{~mm} ; F_{1}=11.7$ and $Z_{\text {in }}=228 \Omega$.

Figure 2 shows the reflection and transmission characteristics of the optimized antenna. It can be observed, that the $\mathrm{TM}_{200}$ mode has significantly higher quality factor than the $\mathrm{TM}_{100}$ one. Despite of this, the achieved bandwidths for both modes are comparable: $143 \mathrm{MHz}$ for $S_{11}$ and $100 \mathrm{MHz}$ for $S_{22}$. Isolation is better than $19 \mathrm{~dB}$ within the entire band of interest.

Figure 3 shows radiation patterns for the both modes. Excitation at port 1 produces a unidirectional beam with realized gain of $7.2 \mathrm{dBi}$. Excitation at port 2 produces radiation directed sideway with maximum realized gain of $4.6 \mathrm{dBi}$.

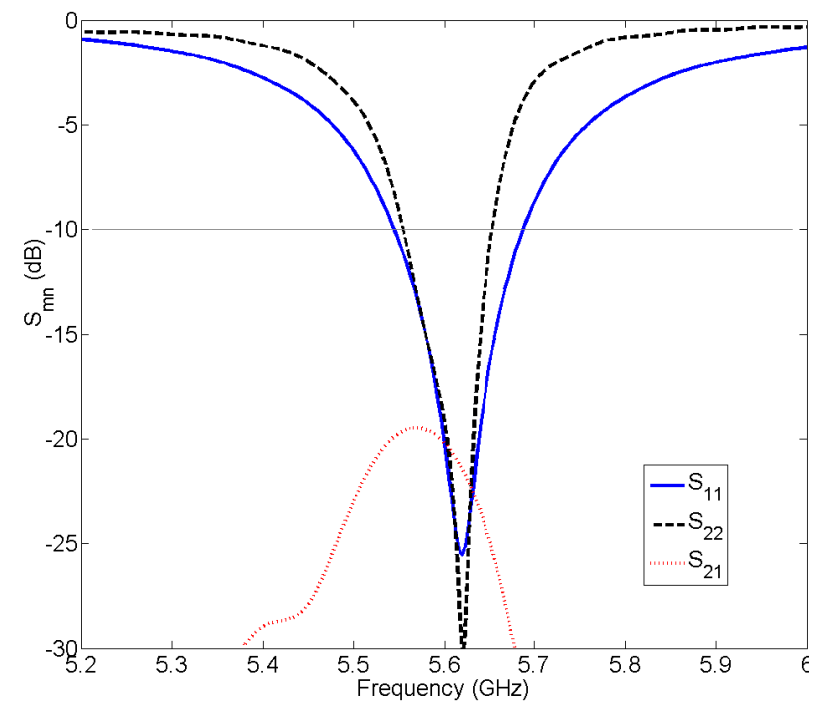

Fig. 2. Simulated reflection and transmission characteristics of the proposed antenna.

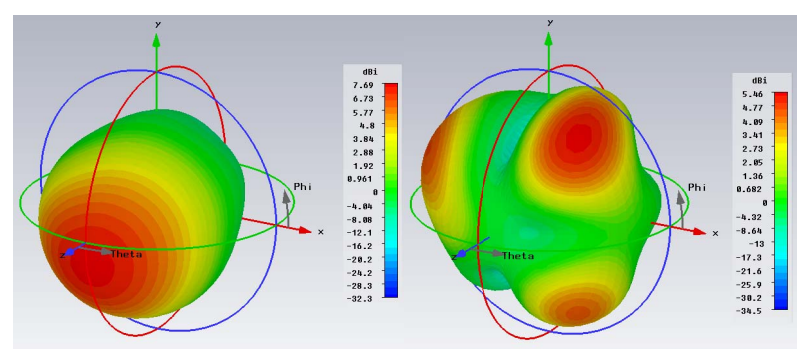

(a)

(b)

Fig. 3. Simulated radiation patterns of the optimized antenna at $5.6 \mathrm{GHz}$ : (a) port 1 ; (b) port 2.

\section{ACKNOWLEDGEMENT}

This work was supported by "ELEVATE: Irish Research Council International Career Development Fellowship - cofunded by Marie Cure Actions," grant number: ELEVATEPD/2014/79.

\section{REFERENCES}

[1] D. Heberling, and C. Oikonomopoulos-Zachos, "On multiport antennas for MIMO-systems," Proc. International Conference on Microwave Radar and Wireless Communications, MIKON 2010, Vilnius, Lithuania, 14-16 June 2010.

[2] A. Narbudowicz, X. L. Bao, and M. J. Ammann, "Omnidirectional microstrip patch antenna with reconfigurable pattern and polarisation," IET Microwaves, Antennas \& Propagation, vol. 8, no. 11, pp. 872-877, Aug. 2014.

[3] E. R. Iglesias, O. Q. Teruel, and M. S. Ferflifldez, "Compact multimode patch antennas for MIMO applications," IEEE Antennas Propag. Mag., vol. 50, no. 2, pp. 197-205, Apr. 2008.

[4] S. Koziel, J.W. Bandler and K. Madsen, "Towards a rigorous formulation of the space mapping technique for engineering design," Proc. Int. Symp. Circuits, Syst., ISCAS, vol. 1, 2005, pp. 5605-5608.

[5] S. Koziel, "Computationally efficient multi-fidelity multi-grid design optimization of microwave structures," Applied Comput. Electrom. Soc. J., vol. 25, no. 7, pp. 578-586, 2010. 